cond-mat/9303027

NSF-ITP-93-25

\title{
Method for Measuring the Momentum-Dependent Relative Phase of the Superconducting Gap of High-Temperature Superconductors
}

\author{
M.E. Flatté \\ Institute for Theoretical Physics \\ University of California, Santa Barbara, CA 93106-4030 \\ S. Quinlan and D.J. Scalapino \\ Physics Department \\ University of California, Santa Barbara, CA 93106-9530
}

\begin{abstract}
The phase variation of the superconducting gap over the (normal) Fermi surface of the high-temperature superconductors remains a significant unresolved question. Is the phase of the gap constant, does it change sign, or is it perhaps complex? A detailed answer to this question would provide important constraints on various pairing mechanisms. Here we propose a new method for measuring the relative gap phase on the Fermi surface which is direct, is angle-resolved, and probes the bulk. The required experiments involve measuring phonon linewidths in the normal and superconducting state, with resolution available in current facilities. We primarily address the $\mathrm{La}_{1.85} \mathrm{Sr}_{.15} \mathrm{CuO}_{4}$ material, but also propose a more detailed study of a specific phonon in $\mathrm{Bi}_{2} \mathrm{Sr}_{2} \mathrm{CaCu}_{2} \mathrm{O}_{8}$.
\end{abstract}

74.25.Kc, 74.72.Dn, 74.72.Hs

Typeset Using REVTEX 
The momentum and frequency dependence of the superconducting gap reflects the underlying structure of the pairing interaction. In traditional low-temperature superconductors the gap depends weakly on momentum with negligible anisotropy on the (normal) Fermi surface, and exhibits a clear frequency dependence near important phonon energies. This implies that the effective pairing interaction is short range in space, retarded in time, and relatively isotropic. Detailed analysis of the frequency dependence of the gap determined from $I(V)$ measurements provides the most direct evidence that the electron-phonon interaction [1] is responsible for superconductivity in these materials. The $p$-wave gap in the ${ }^{3} \mathrm{He}$ superfluids [2] and the evidence of nodes [3] consistent with $d$-wave pairing in some of the heavy fermion superconductors suggest that the basic interactions in these systems repel at momentum transfers comparable to the Fermi momentum. The exchanges of paramagnetic [4] or antiparamagnetic [5] spin-fluctuations have been proposed as models for such interactions.

A variety of gap symmetries have been suggested for the cuprate-oxide superconductors based on particular models of the pairing mechanism. Phonon and charge fluctuation mediated mechanisms typically lead to gaps which may vary in magnitude but remain constant in phase over the Fermi surface. Here we will call such gaps anisotropic s-wave gaps. Pairing interactions associated with the exchange of an antiferromagnetic spin-fluctuation in the Hubbard model produce a $d_{x^{2}-y^{2}}$ gap which changes sign on the Fermi surface [6,7]. Slave boson approximations and variational calculations for the $t-J$ model have led to $d_{x^{2}-y^{2}}$ and complex d-wave gaps [8]. One example of a complex $d$-wave gap has a constant magnitude, but a phase which varies as $\exp (2 i \phi)$, where $\phi$ is the angular location of a point on the Fermi surface. Clearly, a method for determining the relative phase of the gap between different points on the Fermi surface would substantially constrain any theory of high-temperature superconductivity. Here we show how neutron scattering measurements of phonon linewidths can provide such a probe.

This method involves locating and measuring anomalies (discontinuities) in phonon linewidths which occur as a function of temperature or frequency at the quasiparticle-pair- 
production threshold. Previously a method was proposed [9] for measuring the angle-resolved gap magnitude using only the threshold frequencies. That method has been applied [10] to recent data [11] on a phonon in $\mathrm{Bi}_{2} \mathrm{Sr}_{2} \mathrm{CaCu}_{2} \mathrm{O}_{8}$. Now we demonstrate that measuring the threshold discontinuity size (in addition to its onset frequency) extends that method to produce angle-resolved measurements of the relative gap phase between two points on the Fermi surface.

Other methods used to detect the existence of $d$-wave gaps search for pronounced gap magnitude anisotropy. The temperature-dependence of various thermodynamic quantities may contain anomalous (power-law) contributions from gap nodes. A direct probe of gap magnitude, angle-resolved photoemission, measures the gap magnitude around the Fermi surface. Rather than relying on gap magnitude nodes to indicate changes in the gap phase, our method directly determines the relative phase of the gap, $\Delta \phi$, between different points on the Fermi surface. This type of measurement is crucial for theory because, even if the gap magnitude is isotropic, the phase could make it $d$-wave, such as for the gap function $\exp (2 i \phi)$. Conversely, gap nodes do not guarantee gap sign changes. The method we propose has adequate Fermi surface angular resolution and, most importantly, probes the bulk properties of the superconductor.

The method for measuring the energy-gap magnitude in Ref. [9] took advantage of the following geometrical arguments. For phonon momenta larger than an inverse coherence length, the two quasiparticles produced from a phonon's decay appear at only two places on a quasi-two-dimensional Fermi surface. Fig. 11 shows this geometry. Both the head and the tail of the phonon wavevector (two are shown, vectors $\mathbf{q}_{\mathbf{1}}$ and $\mathbf{q}_{\mathbf{2}}$ ) must lie on the Fermi surface. The quasiparticles are created at the head of this vector, and at the Fermi surface point opposite the tail (as shown in Fig. 1 for $\mathbf{q}_{\mathbf{2}}$, where the two points are labeled $\mathbf{k}_{\mathbf{o}}$ and $\mathbf{q}_{\mathbf{2}}-\mathbf{k}_{\mathbf{o}}$ ). Any other placement of the phonon wavevector results in an energy cost from creating quasiparticles off the Fermi surface which far exceeds the energy of a characteristic phonon. The minimum energy for a phonon to decay into two quasiparticles is thus a function of momentum. This energy constraint defines a surface in $(\mathbf{q}, \omega)$ space called the 
threshold surface.

A derivation similar to that in previous work on three-dimensional superconductors with isotropic gaps [12] identifies a discontinuity in the linewidth of a phonon when it crosses the threshold surface. A crossing can occur either through variation of the phonon frequency and momentum (moving along a dispersion curve) or altering the threshold surface (changing the temperature). In order to determine the gap magnitude, the size of the discontinuity is unimportant, so long as it is observable.

We show in this Letter that the size of the discontinuity yields a direct measurement of the phase of the gap. For a given phonon, the ratio of the discontinuity at low temperature to the normal-state linewidth is

$$
\frac{\Delta \gamma}{\gamma_{N}}=\frac{\pi}{2} \cos ^{2}\left(\frac{\Delta \phi}{2}\right)
$$

where $\Delta \phi$ is the relative phase between gaps at the locations where quasiparticles are created (described above). To understand this, we note that the phonon linewidth is proportional to the imaginary part of the density-density response function [13]

$$
\begin{aligned}
& \operatorname{Im} P(\mathbf{q}, \omega)= \\
& \frac{\pi}{N} \sum_{\mathbf{k}}\left\{\left[1+\frac{\varepsilon_{\mathbf{q}-\mathbf{k}} \varepsilon_{\mathbf{k}}-\operatorname{Re}\left(\Delta_{\mathbf{k}} \Delta_{\mathbf{q}-\mathbf{k}}^{*}\right.}{E_{\mathbf{q}-\mathbf{k}} E_{\mathbf{k}}}\right]\left(f\left(E_{\mathbf{q}-\mathbf{k}}\right)-f\left(E_{\mathbf{k}}\right)\right) \delta\left(\omega-E_{\mathbf{q}-\mathbf{k}}+E_{\mathbf{k}}\right)\right. \\
& \left.\quad+\frac{1}{2}\left[1-\frac{\varepsilon_{\mathbf{q}-\mathbf{k}} \varepsilon_{\mathbf{k}}-\operatorname{Re}\left(\Delta_{\mathbf{k}} \Delta_{\mathbf{q}-\mathbf{k}}^{*}\right)}{E_{\mathbf{q}-\mathbf{k}} E_{\mathbf{k}}}\right]\left(f\left(E_{\mathbf{q}-\mathbf{k}}\right)+f\left(E_{\mathbf{k}}\right)-1\right) \delta\left(\omega-E_{\mathbf{q}-\mathbf{k}}-E_{\mathbf{k}}\right)\right\} .
\end{aligned}
$$

Here $\varepsilon_{k}$ is the normal state dispersion relation for electrons (measured from the chemical potential), $f(\varepsilon)$ is the Fermi function, $\Delta_{\mathbf{k}}$ is the (momentum-dependent) gap, and $E_{\mathbf{k}}=$ $\sqrt{\varepsilon_{\mathbf{k}}^{2}+\Delta_{\mathbf{k}}^{2}}$. The first term in Eq. 2 arises from the scattering of thermal quasi-particles, and the second term corresponds to quasiparticle pair production. The square brackets contain the usual BCS coherence factors. When the phonon energy exceeds the minimum energy $\left|\Delta_{\mathbf{k}_{\mathbf{o}}}\right|+\left|\Delta_{\mathbf{q}-\mathbf{k}_{\mathbf{o}}}\right|$, pair production of quasiparticles occurs. The discontinuity in the linewidth arises due to the square-root divergence in the density of states near each of the points $\mathbf{k}_{\mathbf{o}}$ and $\mathbf{q}-\mathbf{k}_{\mathbf{o}}$. The coherence factor regulates the magnitude of the discontinuity. When both 
quasiparticles are created on the Fermi surface, the coherence factor reduces to this simple form [14]:

$$
\frac{1}{2}\left(1+\frac{\operatorname{Re}\left[\Delta_{\mathbf{k}_{\mathbf{o}}} \Delta_{\mathbf{q}-\mathbf{k}_{\mathbf{o}}}^{*}\right]}{\left|\Delta_{\mathbf{k}_{\mathbf{o}}}\right|\left|\Delta_{\mathbf{q}-\mathbf{k}_{\mathbf{o}}}\right|}\right)=\cos ^{2}\left(\frac{\Delta \phi}{2}\right)
$$

We now consider two different phonons in $\mathrm{La}_{1.85} \mathrm{Sr}_{.15} \mathrm{CuO}_{4}$, shown as $\mathbf{q}_{\mathbf{1}}$ and $\mathbf{q}_{\mathbf{2}}$ on the Fermi surface [15] (Fig. 11). We will examine their behavior for four different gap functions:

i. isotropic.

$$
\Delta_{\mathbf{k}}=\Delta(T)
$$

We choose $T_{c}=40 K$ and $\Delta(T)$ to follow the BCS temperature dependence with $2 \Delta(0)=$ $6 k_{B} T_{c}$. The minimum energy of decay for phonons in this superconductor is not momentum dependent.

ii. constant phase with nodes.

$$
\Delta_{\mathrm{k}}=\frac{\Delta(T)}{2}\left|\cos \left(k_{x} a\right)-\cos \left(k_{y} a\right)\right|
$$

has nodes on the Fermi surface at the four locations indicated with black squares on Fig. 1. This is a limiting example of an anisotropic s-wave gap.

iii. $d_{x^{2}-y^{2}}$.

$$
\Delta_{\mathbf{k}}=\frac{\Delta(T)}{2}\left\{\cos \left(k_{x} a\right)-\cos \left(k_{y} a\right)\right\}
$$

has the same gap magnitude as the above anisotropic s-wave gap, and thus is indistinguishable from it in most probes. However, the relative phase $\Delta \phi$ can take two values: 0 and $\pi$. The former case occurs for phonon $\mathbf{q}_{\mathbf{1}}$, while the latter for $\mathbf{q}_{\mathbf{2}}$.

iv. complex d-wave.

$$
\Delta_{\mathbf{k}}=\Delta(T) \exp (2 i \phi)
$$

has the same gap magnitude as the isotropic case, and so is indistinguishable from it for most probes. The relative phase between two points, however, can take on a continuum of 
values from 0 to $\pi$. We will focus on the above four gaps in this Letter although the theory applies to all gap functions.

The ratio of the linewidth at a low temperature $\left(T_{c} / 2\right)$ to that in the normal state is shown for all four gaps for phonon $\mathbf{q}_{1}=(0.4 \pi, 0)$ in Fig. 2 and $\mathbf{q}_{2}=(\pi, 0.5 \pi)$ in Fig. 3 . This ratio depends on the imaginary part of the polarization (density-density) response according to the following equation:

$$
\frac{\gamma\left(\mathbf{q}, \omega T_{c} / 2\right)}{\gamma\left(\mathbf{q}, \omega, T_{c}\right)}=\frac{\operatorname{Im} P\left(\mathbf{q}, \omega, T_{c} / 2\right)}{\operatorname{Im} P\left(\mathbf{q}, \omega, T_{c}\right)}
$$

We find this ratio convenient because it does not depend on the electron-phonon coupling.

There are two discontinuities in the linewidths for the anisotropic s-wave and $d_{x^{2}-y^{2}}$ gaps in Fig. 2 because there are two possible ways to create quasiparticles on the Fermi surface: the normal process, labeled by $\mathbf{q}_{\mathbf{1}}$ in Fig. 1, and an Umklapp process, the dotted vector on Fig. 1. Since the gap magnitudes in these regions differ, the discontinuities occur at different energies. The relative phase for these two gap functions is identical for both channels, so the linewidths are indistinguishable on Fig. 2.

There is a single discontinuity for the isotropic-gap case because both of these decay channels have the same minimum energy, $2 \Delta(T)$. For the complex d-wave gap, the gap magnitudes for these two decay channels are the same, but the phase of the gap at $\mathbf{k}_{\mathbf{o}}$ and $\mathbf{q}_{\mathbf{1}}-\mathbf{k}_{\mathbf{o}}$ differ. For the normal case, the phase difference $\Delta \phi=.35 \pi$, while for the Umklapp case, $\Delta \phi=.22 \pi$. These relative phases yield discontinuities of $73 \%$ and $88 \%$ respectively of the isotropic-gap result. Since they occur at the same energy, the observed discontinuity has an intermediate size of $\sim 80 \%$, as found in Fig. 2.

For the momentum transfer $\mathbf{q}_{2}$, shown in Fig. 3 there is only one decay channel, so there is a single discontinuity for all four gap functions. For the constant phase gaps, (i) and (ii), this discontinuity has its maximum size. For the $d_{x^{2}-y^{2}}$ gap, the relative phase between $\mathbf{k}_{\mathbf{o}}$ and $\mathbf{q}_{2}$ is $\pi$, so there is no step in the linewidth. There is merely a discontinuity in the slope of the linewidth. For the complex d-wave gap, the relative phase is quite close to $\pi$, and the size of the discontinuity reaches merely $2 \%$ of maximum. It is clear in Figs. 2 and 3 that 
not only are superconductors with different gap magnitudes distinguishable from each other (isotropic from anisotropic s-wave), but those which only differ by their gap phase as well ( $d_{x^{2}-y^{2}}$ from anisotropic s-wave and complex d-wave from isotropic).

Figs. 4 and 5 shows the temperature-dependence of phonons with momenta $\mathbf{q}_{\mathbf{1}}$ and $\mathbf{q}_{\mathbf{2}}$, respectively, and energy $\omega=\Delta(0)$ in the four superconductors. Although the energies of phonons with momentum $\mathbf{q}_{2}$ have not been measured, a candidate phonon should exist [16] for a large range of values of $\Delta(0)$. There are three known candidates for $\mathbf{q}_{\mathbf{1}}$.

In Fig. 4 all of the superconductors have an enhanced linewidth below the critical temperature $\left(T_{c}\right)$, and the discontinuities occur at a temperature resolvable from $T_{c}$. For the gap functions with anisotropic gap magnitudes, the two decay channels are resolvable as separate discontinuities. A linewidth enhancement at temperatures just above the discontinuity, similar to those shown, was observed in several phonons in a classic niobium experiment [17]. The enhancement for the complex d-wave gap is lower than the isotropic one because the relative phase between $\mathbf{k}_{\mathbf{o}}$ and $\mathbf{q}_{\mathbf{1}}-\mathbf{k}_{\mathbf{o}}$ differs from 0 . From Eq. 1, there will be an enhancement before the discontinuity unless the relative phase exceeds $.41 \pi$.

A relative phase of $\pi$ between $\mathbf{k}_{\mathbf{o}}$ and $\mathbf{q}_{\mathbf{2}}-\mathbf{k}_{\mathbf{o}}$ in Fig. 5 for the $d_{x^{2}-y^{2}}$ case causes the linewidth to monotonically decrease from $T_{c}$ without a discontinuity. For the complex d-wave, the relative phase is close to $\pi$, causing the linewidth at $T_{c}$ to drop, and the discontinuity at low temperature to be extremely small. Thus the discontinuity size, and the slope of the linewidth at $T_{c}$ probe the relative phase of the gap.

Recently Mook et al. [11] reported the narrowing of a low-energy phonon in $\mathrm{Bi}_{2} \mathrm{Sr}_{2} \mathrm{CaCu}_{2} \mathrm{O}_{8}$ upon cooling through $T_{c}$. The resolution of that measurement was inadequate to determine the structure around the discontinuity. However, performing the measurement at a slightly higher resolution should determine the nature of that structure. The enhancement prior to the discontinuity expected for $s$-wave gaps would not be expected for this phonon if the superconductor had a $d_{x^{2}-y^{2}}$ gap.

Other electronic contributions to a phonon's decay rate besides the pair processes could complicate the interpretation of results. We have described how, if the relative phase is 
small, for temperatures slightly above the discontinuity the linewidth will increase as the temperature drops. If, however, the relative phase is close to $\pi$, the linewidth decreases just before the discontinuity. Since other contributions to the linewidth are expected to be smooth near the pair-production threshold, even if those other contributions are large, the temperature dependence of the linewidth just above the discontinuity will retain this strong dependence on the relative gap phase.

The probe described in this Letter provides a direct, angle-resolved measurement of the relative gap phase in high-temperature superconductors' bulk. Such measurements can constrain the range of acceptable theories of high-temperature superconductors.

We thank B.-H. Schüttler for suggesting an effective way of numerically calculating the polarizability. S.Q. and D.J.S. acknowledge the support of the National Science Foundation grant DMR90-02492. M.E.F. acknowledges the support of this work by the National Science Foundation under Grant No. PHY89-04035. The numerical calculations reported in this paper were performed at the San Diego Supercomputer Center. 


\section{REFERENCES}

[1] J. Rowell, W. McMillan, Superconductivity I, (R.D. Parks, ed.), Marcel Dekker, New York, 1969.

[2] A.J. Leggett, Rev. Mod. Phys. 47, 331 (1975).

[3] C. Broholm, et al., Phys. Rev. Lett. 65, 2062 (1990).

[4] P.W. Anderson, W.F. Brinkman, Phys. Rev. Lett. 30, 1108 (1973).

[5] K. Miyake, S. Schmitt-Rink, C.M. Varma, Phys. Rev. B 34, 6554 (1986). D.J. Scalapino, E. Loh, J. Hirsch, Phys. Rev. B 34, 8190 (1986).

[6] N.E. Bickers, D.J. Scalapino, R.T. Scalettar, International Journal of Modern Physics B 1, 687 (1987); N.E. Bickers, D.J. Scalapino, S.R. White, Phys. Rev. 62, 961 (1989).

[7] P. Monthoux, A. Balatsky, D. Pines, Phys. Rev. Lett. 67, 3448 (1991); P. Monthoux, D. Pines, Phys. Rev. Lett. 69, 961 (1992).

[8] In addition to the $d_{x^{2}-y^{2}}$ gap, an $s+i d$ gap is derived in G. Kotliar, J. Liu, Phys. Rev. B 38, 5142 (1988). G. Kotliar, Phys. Rev. B 37, 3664 (1988). An $s+i d$ gap is also suggested in G.J. Chen, R. Joynt, F.C. Zhang, C. Gross, Phys. Rev. B 42, 2662 (1990). Another complex d-wave gap is $d_{x^{2}-y^{2}}+i \alpha d_{x y}$, proposed by R. Laughlin (preprint).

[9] M.E. Flatté, Phys. Rev. Lett. 70, 658 (1993).

[10] M.E. Flatté, NSF-ITP-93-16, to be published.

[11] H.A. Mook, et al., Phys. Rev. Lett. 69, 2272 (1992).

[12] V.M. Bobetic, Phys. Rev. 136, A1535 (1964). H.G. Schuster, Solid State Communications 13, 1559 (1973).

[13] Numerical calculations of $P(\mathbf{q}, \omega)$ for the Hubbard model with $U / t=4$ find that $\operatorname{Im} P(\mathbf{q}, \omega)$ is proportional to the $U=0$ result when $\omega / t$ is small (N. Bulut, private 
communication).

[14] In magnetic scattering of neutrons, the cross-section is proportional to the imaginary part of the magnetic susceptibility. In this case, the coherence factor for pair-production is altered to become $\sin ^{2}(\Delta \phi / 2)$, and no discontinuity exists if the gap phase is constant. A jump will occur for a $d_{x^{2}-y^{2}}$ gap (N. Bulut, D.J. Scalapino, Phys. Rev. B, in press). An important limitation for magnetic scattering, however, is that the resonant coupling of the quasiparticles to the neutrons is through spin fluctuations rather than phonons. In contrast to the wide availability in momentum and energy of phonons in the high-temperature materials (due to the large unit cells), spin fluctuations only exist near $(\pi, \pi)$.

[15] M.S. Hybertsen, et al., Phys. Rev. B 41, 11068 (1990).

[16] L. Pintschovius, et al., International Seminar on High-Temperature Superconductivity, ed. V.L. Aksenov, World Scientific, 1990.

[17] S.M. Shapiro, G. Shirane, J.D. Axe, Phys. Rev. B 12, 4899 (1975). 


\section{FIGURES}

FIG. 1. Fermi surface of $\mathrm{La}_{1.85} \mathrm{Sr}_{.15} \mathrm{CuO}_{4}$, taken from the parametrization of Ref. [15]. Nodes in the $d_{x^{2}-y^{2}}$ gap function occur on the Fermi surface at the black squares. The sign of the $d_{x^{2}-y^{2}}$ gap is indicated on the Fermi surface. This Letter examines in detail phonons with momenta $\mathbf{q}_{\mathbf{1}}=(0.4 \pi, 0)$ and $\mathbf{q}_{\mathbf{2}}=(\pi, 0.5 \pi)$. The two quasiparticles created by the decay of phonon $\mathbf{q}_{\mathbf{2}}$ form at locations $\mathbf{k}_{\mathbf{o}}$ and $\mathbf{q}_{\mathbf{2}}-\mathbf{k}_{\mathbf{o}}$ on the Fermi surface. There are two decay channels for phonon $\mathbf{q}_{\mathbf{1}}$, the normal, labeled $\mathbf{q}_{\mathbf{1}}$, and Umklapp (dotted vector).

FIG. 2. The linewidth of phonons with momentum $\mathbf{q}_{\mathbf{1}}$ as a function of frequency for four different gaps. In the remaining figures, results for these four cases will be plotted as follows: isotropic (solid), anisotropic s-wave (dashed), $d_{x^{2}-y^{2}}$ (dotted), and complex d-wave (dot-dash). In this figure, the curve for the $d_{x^{2}-y^{2}}$ gap lies on top of the anisotropic s-wave curve. The abscissa is normalized to the normal linewidth at $T_{c}$.

FIG. 3. As in Fig. 2, but for momentum $\mathbf{q}_{2}$. In contrast to Fig. 2, here the $d_{x^{2}-y^{2}}$ curve is distinguishable from the anisotropic s-wave.

FIG. 4. The linewidth of a phonon with momentum $\mathbf{q}_{\mathbf{1}}$ and energy $\Delta(0)$ as a function of temperature for the four different gaps. As in Fig. 2, the dotted line $\left(d_{x^{2}-y^{2}}\right.$ gap $)$ is not visible, since it lies on top of the dashed line (anisotropic s-wave).

FIG. 5. As in Fig. \& but for momentum $\mathbf{q}_{2}$. Here the $d_{x^{2}-y^{2}}$ curve is substantially different from the anisotropic s-wave curve. 